\title{
El movimiento de vida independiente en España
}

\section{The independent living movement in Spain}

\section{Palabras clave}

Movimiento de vida independiente, centro de vida independiente, asistente personal, consejo inter pares, autonomía, derechos humanos.

\section{Keywords}

Independent life movement, independent living center, personal assistant, peer council, autonomy, human rights.
1. Introducción: los modelos de aproximación a la discapacidad

Básicamente, estos modelos pueden reducirse a tres: prescindencia, rehabilitador y social (Palacios, 2008: 25). El primero de ellos es el multisecular de abandono y marginación, solo superado tras las guerras mundiales, cuando la presencia de una gran cantidad de personas mutiladas a las que no se podía adjudicar su situación a diversas supersticiones, derivó en que se las aceptara como víctimas a las que había que apoyar médicamente y rehabilitar para vivir en sociedad en la medida de lo posible. Este segundo modelo, aunque constituyó un gran paso, era netamente asistencial de supervivencia. La institucionalización es el fenómeno característico de este momento, en el caso de que las familias no pudieran hacerse cargo de los cuidados. La actitud es paternalista y asistencial ante una diversidad que se considera como deficiencia, atribuyendo a la persona que la 'padece' un valor inferior al considerado 'normal' en una sociedad capacitista (ableism) (Toboso, 2017: 73-81).

El gran paso se da con el cambio al modelo social. Este modelo surge a finales de los años sesenta y principios de los setenta del siglo pasado, en la cultura anglosajona, entre Estados Unidos y Reino Unido. De Estados Unidos toma su tradición de campañas basadas en los derechos civiles,

\author{
Gonzalo José Carbonell \\ Aparici \\ $<$ maitreya.gonzalo@gmail.com> \\ COCEMFE Valencia. España
}

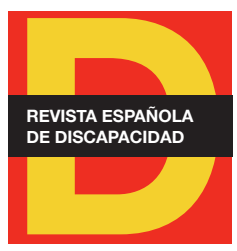

Para citar:

Carbonell, G. J. (2019): "El movimiento de vida independiente en España". Revista Española de Discapacidad, 7 (II): 201-214.

Doi: <https://doi.org/10.5569/23405104.07.02.12> 
de acciones políticas de masas, de libertad política y económica; es el que adopta el movimiento de vida independiente (MVI). En el Reino Unido, desde un enfoque diferente, el MVI se concentró en alcanzar cambios sociopolíticos en relación con una legislación respetuosa con los derechos humanos (en la línea de los posteriormente llamados derechos sociales). EI MVI puede considerarse como el antecedente inmediato del nacimiento del modelo social, que luego siguió su propio rumbo, atribuido a Vic Finkelstein en el Reino Unido (Finkelstein, 2001).

\section{Origen y expansión del movimiento de vida independiente}

Hay consenso en situar cronológicamente el nacimiento del movimiento de vida independiente en 1962, el día en que Edward Verne Roberts (1939-1995) ingresa en la Universidad de Berkeley, California (García, 2003). Ed Roberts era una persona con diversidad funcional 'severa' que desde su infancia se empeñó en llevar una vida 'normalizada' (Palacios y Romañach, 2006: 52-53).

En aquel entonces había en Estados Unidos pocas universidades con accesibilidad para veteranos de guerra, pero Ed eligió la que le pareció mejor para su proyecto académico de licenciatura en Ciencias Políticas. La Universidad de Berkeley no tenía accesibilidad adecuada para él. Tuvo que instalarse en la enfermería y, para sus necesidades personales, contrató el apoyo de asistentes personales. Siguiendo su ejemplo, en 1967 había ya 12 estudiantes con diversidad funcional que vivían con él en la enfermería.

En 1970 inaugura un programa para estudiantes con diversidad funcional física, diseñado por los propios interesados en base a sus experiencias personales, contando con una oficina accesible en el campus. A partir de aquí surge la idea de implantar un programa similar fuera del ámbito universitario, de modo que las personas con diversidad funcional pudieran vivir independientemente y de forma integrada en sus comunidades: se gesta la idea de centro de vida independiente (CVI, CIL o ILC en inglés), que se inaugura en Berkeley en 1972.

En este sencillo proceso se puso en marcha un potente armazón de ideas sobre la vida independiente, donde Roberts redefine el concepto de independencia como el "control sobre la propia vida" y, por otra parte, se empiezan a trazar las líneas esenciales del MVI: auto-organización, consejo inter pares, asistencia personal remunerada, accesibilidad universal e inserción comunitaria (Palacios y Romañach, 2006: 54-55).

Esta experiencia originaria se difundió rápidamente, abierta a todo tipo de diversidad y de cultura, afrontando el tema de las discriminaciones múltiples. A finales de los años setenta del siglo XX, se forman grupos inspirados por los principios de vida independiente en otros países, realizando el primer Congreso Internacional Mundial (1980) en Winnipeg (Canadá), y fundando allí la primera organización internacional de todo tipo de discapacidad con el nombre de Disabled People's International (DPI) (Martínez, 2003).

De esta expansión, queremos resaltar la importancia que tuvo el hecho de que la sensibilidad civilista de Estados Unidos se reforzara con la sensibilidad social de algunos lugares estratégicos tales como el Reino 
Unido, cuyo líder V. Finkelstein conoció in situ la experiencia de Berkeley, como punto clave en la iniciación del modelo social. En el caso de Suecia, A. Ratzka también se enriqueció en Berkeley con la experiencia del $\mathrm{MVI}$, siendo otro punto de referencia en el desarrollo europeo de la visión social de los derechos de las personas con discapacidad (Ratzka, A. D., 2003, 1992, 1982). Noruega, en la misma línea sueca, ha dado ejemplo con una única cooperativa a nivel estatal (ULOBA), que ha sabido combinar perfectamente la colaboración del Estado con las libertades pedidas desde el MVI (Maraña, 2004: 92-97; Moreno y Del Pino, 2015).

Más allá de Europa, Japón ha tenido también un importante papel como punto de conexión entre Berkeley y el continente asiático (Nakanishi, 1997; Hayashi y Okuhira, 2008). En general, desde el MVI, se han apoyado económicamente proyectos de vida independiente en países menos desarrollados, sobre todo desde la Red europea de vida independiente (ENIL), que ha sido un motor muy importante en la expansión del movimiento (Gardien, 2010).

3. Los conceptos fundamentales del movimiento de vida independiente

a. Reivindicación de derechos, desde los derechos humanos hasta el último de los derechos civiles o sociales, en base a un principio básico de igualdad, mediante medidas de acción positiva dirigidas hacia los colectivos especialmente vulnerables. Fue fuente de inspiración para la Convención sobre los derechos de las personas con discapacidad (Naciones Unidas, 2006). Este tratado vino a ratificar el modelo social de la discapacidad y el derecho a la autonomía personal, incluyendo el derecho a la asistencia personal en su artículo 19. A partir de entonces, dicha Convención ha sido la bandera reivindicativa del MVI que, años más tarde, ha sido ratificada por la Observación general núm. 5 (2017) sobre el derecho a vivir de forma independiente y a ser incluido en la comunidad. Esta Convención es la base de toda la normativa europea posterior a 2006. Se trata de crear una igualdad de oportunidades, sentando las bases para un derecho fundamental (López y Gutiérrez, 2002: 51, 56 y 102).

b. La pretensión de igualdad surge de la concepción de la discapacidad como un elemento más de la diversidad humana.

c. El derecho a la igualdad de oportunidades implica derecho a la no discriminación por la diversidad funcional. Esta correlación nos lleva a otra de las grandes reivindicaciones del MVI: la accesibilidad universal (De Asís, 2016), imprescindible para poder disfrutar un derecho efectivo (Miranda, 2016).

d. Consecuencia directa de la dignidad de la persona es la participación como síntoma de empoderamiento: "Nada sobre nosotros sin nosotros". La diversidad funcional no implica incapacidad de obrar y decidir, que en un Estado de derecho solo puede decidir un juez (Lasarte, 2010: 52-69).

e. La finalidad de estos presupuestos (igualdad, accesibilidad, empoderamiento) es la consecución de una vida lo más independiente posible, a la que cualquier persona debe poder aspirar (Urmeneta, 2012: 59; Shakespeare, 2008). La reivindicación fundamental se centra en la libertad real de elección.

f. Los grupos de autoayuda son una herramienta muy utilizada por colectivos cuyos miembros afrontan individualmente dificultades especiales. Su poder radica fundamentalmente en aprovechar la experiencia 
de las personas que están en circunstancias parecidas. Ha dado muestra de eficacia en diferentes contextos en combinación con otras herramientas. Una aportación importante del MVI ha sido la consideración de esta gestión no solo a nivel individual, sino también a nivel colectivo, asumiendo la formación y dirección de los centros de vida independiente con elaboración de programas, estudios y proyectos tanto individuales como grupales, donde no se excluye la participación de otras personas, pero sin que estas lleven en exclusiva la dirección.

g. La otra gran herramienta básica son las ayudas técnicas, consideradas en su conjunto, que complementan los necesarios cambios de diseño en favor de la accesibilidad en muchos campos tecnológicos en constante innovación: internet, transporte y aparatos facilitadores de todo tipo, que vienen a sumarse a otros apoyos institucionales como programas, subvenciones y prestaciones de servicios o económicas. Todas ellas contribuyen a la vida independiente y el criterio básico de aceptación por parte del MVI será el índice de protagonismo que se concede a las personas con diversidad funcional en su proyección y diseño, así como su utilidad real para su autonomía (Centeno et al., 2008).

h. Entre estos apoyos el MVI destaca la asistencia personal, que completa y coordina todos los demás, bajo la supervisión del usuario con diversidad funcional. El asistente personal no es un cuidador, sino una herramienta básica para la independencia de la persona con diversidad funcional, cuya existencia incide directamente en el cambio de paradigma de atención familiar. No habría que considerar "gastos en dependencia", sino "inversiones en autonomía", implicando retornos sociales, menores costes de oportunidad para cuidadores y disminución de la economía sumergida que, a su vez, incide en la discriminación social, en particular de la mujer inmigrante (Jiménez-Martín y Viola, 2017).

\section{El movimiento de vida independiente en España}

\subsection{Orígenes}

Cuando en 1962 comienza el MVI en los Estados Unidos, en España aún no se había desarrollado una conciencia cívica sobre el modo idóneo de abordar los problemas de la discapacidad. Se trataba con espíritu de beneficencia, a la vez que se intentaba ocultar como estigma. Con mucho retraso respecto a otros países europeos, la Constitución Española de 1978 incluye una referencia explícita a la discapacidad (disminuidos) en el marco de los derechos y deberes fundamentales (art.49) y, a partir de aquí, surgieron asociaciones de personas con diversidad funcional y sus familiares. Aunque en España hubo un fuerte movimiento asociativo, no hay constancia de que en los años ochenta estas asociaciones tuvieran contacto con otras asociaciones europeas o americanas. En 1997, la creación del Comité Español de Representantes de Minusválidos $\left(\right.$ CERMI) ${ }^{1}$ supone la unidad de unas 2000 organizaciones, consiguiendo tener representación unificada ante el Estado y aunar fuerzas en la lucha contra el modelo exclusivamente rehabilitador de la discapacidad.

1. Actualmente Comité Español de Representantes de Personas con Discapacidad. 
En 1982 hubo una primera ley de integración ${ }^{2}$, nunca totalmente implementada, y donde aparecieron las pensiones no contributivas que, junto con otros subsidios y la ampliación del concepto de discapacidad a personas aún con posibilidad activa, supusieron una ayuda significativa para el colectivo. El Estado de las autonomías también supuso diferencias en las situaciones concretas (Maestro y García, 1999).

Al MVI se le etiqueta en un principio como "filosofía americanoide de la discapacidad, radical y segregacionista, solo propia de países ricos" (Maraña y Lobato, 2003: .265). Sin embargo, los criterios del MVI provocaron en todo el mundo grandes progresos para el colectivo, tales como la asistencia personal, el diseño universal y la autogestión. Lo novedoso fue la vuelta al reconocimiento y la primacía de los derechos individuales, sobre las que se basaron responsabilidades sociales.

A España también llegaron pioneros procedentes de Berkeley (García, 2003: 29; Foro de Vida Independiente y Divertad, 2010). Sin embargo, lo importante de estos pioneros fue la creación del Foro de Vida Independiente, verdadero hilo conductor en España del MVI.

El Foro de Vida Independiente y Divertad (FVID) se forma en 2001 con el fin de dar a conocer el MVI. La palabra 'Divertad' es un neologismo del foro que sintetiza 'dignidad' y 'libertad'. Este foro es todo un símbolo del movimiento, donde todos participan en igualdad, sin directivos y sin presupuesto económico. Esta participación directa ha sabido aprovechar la potencia intercomunicadora de internet (Fernández et al., 2016), y le ha permitido ser conocido y participar activamente tanto en la política española como europea a través de ENIL.

\subsection{Las peculiaridades del modelo de vida independiente español}

\subsubsection{Modelo de la diversidad}

El término diversidad funcional se propuso en el FVID de España (Lobato, 2005; Romañach, 2009; Ferreira 2010; Bueno y Villatoro, 2012). Por primera vez se promueve el cambio hacia una terminología no negativa, rompiendo la barrera ellos/nosotros. El término diversidad hace referencia a la diferencia entre unas personas y otras, mientras que la funcionalidad hace referencia a la capacidad de actuar de los seres, distinguiendo entre diferencia orgánica (antes deficiencia) y diversidad funcional (antes discapacidad). El nuevo modelo de la diversidad da un paso más allá del modelo social al construirse sobre el valor de la diversidad y asumiendo la dignidad humana universal como elemento clave de participación y aceptación social. El aspecto fundamental que se pretende denunciar con este modelo es la discriminación (Palacios y Romañach, 2006: 116).

Al hablar de la dignidad como un elemento clave del modelo, se alude a dos vertientes complementarias:

a. Dignidad como valor intrínseco al ser humano o punto de partida: ligado a la vida desde su inicio, independientemente de sus condiciones concretas de existencia, base de los derechos humanos y de la igualdad radical entre las personas.

b. Dignidad como aportación extrínseca o punto de llegada, en relación a la calidad de vida y la efectividad de los derechos: si no está garantizado su ejercicio, no hay calidad de vida, incluso puede llegarse a la

2. España. Ley 13/1982, de 7 de abril, de integración social de los minusválidos (LISMI), Boletín Oficial del Estado, 30 de abril de 1982 , núm. 103, pp. 11106-11112. 
situación de que no merezca la pena vivirla porque no se dan las condiciones necesarias para hacerlo con dignidad.

En este modelo se refuerza el aspecto extrínseco al mismo nivel que el intrínseco, dada la dimensión social de la vida humana. Esta paridad entre ambas vertientes es novedosa y refuerza la importancia de la acción sociopolítica. Usa la bioética como herramienta para conseguir la plena dignidad (Palacios y Romañach, 2006: 190; Sanjosé y Cardona, 2010).

\subsubsection{Modelo de vida independiente}

Aunque Palacios y Romañach (2006: 190) consideran este modelo como integrado en el modelo social y, dentro de él, subsumido en el modelo de la diversidad como su desarrollo más actualizado, consideramos apropiado conservar su entidad como modelo diferenciado. En base al derecho civil y político de libertad de decisión y de control de la propia vida se construyó el MVI. Otra cosa es que, para desarrollar la propia independencia, se necesite cooperación social, así como generar ciertas condiciones sociales, en conexión con los llamados derechos sociales (Pisarelo, 2007; Gargarella, 1998) ${ }^{3}$.

En consecuencia, creemos que el modelo español ha sabido conjugar perfectamente las dimensiones individuales y sociales de la esencia humana, considerando un modelo con tres vertientes: diversidad, independencia y social propiamente dicho. De este modo puede considerarse la persona en todas sus dimensiones, a la vez que exige de la sociedad todos sus derechos, sean individuales o colectivos, sin caer en la falacia de la distinción entre unos derechos políticos y unos derechos sociales de segunda categoría, sometidos a los principios de progresividad y disponibilidad. Separar ambos conceptos pudo ser en su momento un modo pedagógico de avanzar, pero en la nueva conciencia social actual puede convertirse en una excusa para dejar sin garantías unos derechos reconocidos teóricamente por todos.

\subsubsection{Praxis actual}

Los diversos modelos históricos de tratamiento de la discapacidad siguen relativamente vigentes. Por ejemplo, el modelo de prescindencia puede seguir presente al negar sistemáticamente el derecho a nacer de seres humanos con diversidad funcional, al recluirlos en instituciones o en la casa paterna contra su voluntad, al someterlos forzosamente a tratamientos no deseados o a supuestos 'cuidados' que pueden atentar a su dignidad. Hay así reminiscencias de prescindencia en la valoración del aborto eugenésico, en la invisibilización social o en la falta de consideración del colectivo a la hora de diseñar el acceso universal y los programas de atención. Sobre todo, esto sucede a la hora de confeccionar leyes y normativas que les conciernen directamente y que afectan a su calidad de vida (Aparicio, 2016; Honneth, 2010).

Por otro lado, el modelo médico-rehabilitador sigue considerando a la persona con diversidad funcional como plenamente dependiente e inferior en capacidades. Se considera que necesitan cuidados a los que se han

3. Richard Sennet nos recuerda que "la autonomía es una poderosa vía de acceso a la igualdad (...) la dependencia se resolverá en interdependencia. En estas condiciones, la autonomía, en la que reside el respeto al otro, puede concederse libremente (...). Al liberalismo, la dignidad de la dependencia nunca le pareció un proyecto político valioso" (Sennet, 2012: 129 y 132). 
de someter de modo pasivo, en un intento, por lo común inútil, de 'rehabilitar' capacidades que nunca se han tenido, en lugar de apoyar a las personas en el desarrollo de sus capacidades efectivas y en la satisfacción de sus necesidades personales.

Bastida considera como "entendimiento constitucional de la discapacidad", en este sentido, un cambio con el que "de la visión liberal decimonónica del discapaz como individuo con taras, se pasa a la visión republicana del discapaz como ciudadano al que los poderes públicos deben garantizar el ejercicio de sus derechos. En suma, de la creación de status jurídicos de incapacidad se pasa a la identificación de situaciones incapacitantes que el Estado debe remover o paliar y del ocultamiento o ignorancia del discapaz se pasa a su visibilidad social" (Bastida, 2010: 84-85).

Esta consideración política sobre la discapacidad integra la visión del modelo social en su triple vertiente, y también nos introduce en la cuestión de la importancia del contexto sociopolítico para la conformación de los modelos teóricos de aproximación a una misma realidad, con procesos de transformación que se retroalimentan recíprocamente.

\subsection{Oficinas de Vida Independiente ${ }^{4}$}

Siguiendo la trayectoria del MVI se crean centros de vida independiente (CVI), en España llamados Oficinas de vida independiente (OVI), para diferenciarlos de los centros tradicionalmente asistencialistas (Gómez y Arroyo, 2013). Sin embargo, hemos de concluir que su desarrollo es más bien testimonial.

Las más veteranas son OVI Madrid y OVI Barcelona, fundadas ambas en 2006 a partir de un proyecto de vida independiente financiado por la administración pública en Madrid, en donde la OVI queda integrada dentro de la Asociación de parapléjicos y personas con gran discapacidad física de la Comunidad de Madrid (ASPAYM), como uno de sus programas principales. OVI Barcelona está centrada en esta ciudad y participa de fondos tanto administrativos como privados, con la gestión conjunta de varias asociaciones. OVI Galicia se fundó en 2010 como parte de la Oficina de Vida Independiente de Galicia (VIgalicia), asociación nacida en 2007 con apoyo de la administración pública. En sus memorias anuales se pueden ver a cuántas personas han ido atendiendo, la cantidad de horas de asistencia que pueden ofrecer. Los principios por los que se rigen son los generales del MVI. Pero, en los tres casos, de ningún modo alcanzan su independencia total de gestión y queda reducido a un pequeño número de usuarios, del mismo modo que dependen de programas anuales para recibir las ayudas pertinentes.

Actualmente está en formación OVI Canarias y VI Andalucía, que tiene un programa específico para unas pocas personas que funciona como OVI.

4. La información general de estas oficinas se encuentra en las páginas web correspondientes, de donde extraemos lo que nos ha parecido más interesante. 


\subsection{Otras articulaciones y actividades}

Existen también otras asociaciones de vida independiente, como VI Comunidad Valenciana (VICOVAL), Zaragoza Vida Independiente (ZAVI) o el Instituto de Paz, Derechos Humanos y Vida Independiente (IPA$\mathrm{DEVI}$ ), que es una organización sin ánimo de lucro, pionera en España, en la medida en que por primera vez interrelaciona el campo de la paz con el de la diversidad funcional, interpretada desde la Convención Internacional de Derechos Humanos de la ONU y los principios generales de la vida independiente. IPADEVI se convierte en la entidad impulsora de la Declaración Mundial de Compostela 2010 sobre Paz y diversidad funcional (IPADEVI, 2010).

La Federación de Vida Independiente (FEVI) nace con la intención de sumar esfuerzos para alcanzar políticas inclusivas e igualitarias, así como de lograr el cumplimiento efectivo de los derechos individuales y colectivos de las personas con diversidad funcional. El Programa de Vida Independiente desarrollado en Gipuzkoa merece también mención especial, en cuanto que fue el primero llevado a cabo en España en 2004 con sistema de pago directo y libre elección de asistente personal, pero con gestión realizada íntegramente por la administración pública (Urmeneta, 2011).

Tenemos en España el caso relevante de SOLCOM (Asociación para la solidaridad comunitaria de las personas con diversidad). Esta asociación está especializada en denunciar el no cumplimiento de la legislación vigente respecto a la diversidad funcional y renuncia en sus estatutos a las aportaciones financieras de la administración. Apoyada por SOLCOM y el FVID, la web Derechos humanos Ya! se presenta como una herramienta para el seguimiento del respeto a los derechos de las personas con diversidad funcional y el cumplimiento de la aplicación de la Convención de Naciones Unidas.

Otro ejemplo significativo es el de la ONG ¿Cómo quieres vivir?, con servicios de formación, asesoramiento y apoyo a personas con diversidad funcional que desean un cambio en sus vidas, ofreciendo también la posibilidad de formar a voluntarios que apoyen el proceso como asistentes personales. Una particularidad de esta asociación es ofrecer la posibilidad de vivir en primera persona "un día especial", donde una persona con diversidad funcional elige qué quiere hacer realmente un día concreto y lo lleva a cabo comprobando que, con los apoyos necesarios, puede llevar una vida autónoma.

También hay pequeñas empresas formadas en los mismos principios y dirigidas por miembros del FVID, como es el caso de SOLVINDE (Soluciones para una Vida Independiente) en Valencia. Es una pequeña empresa que ofrece toda clase de servicios desde la propia experiencia de la diversidad funcional y los principios del MVI que, en este caso, sirven para satisfacer unas necesidades cotidianas insuficientemente cubiertas por los poderes públicos.

Una característica importante del MVI en España es su importante presencia en la red. Aparte del mencionado FVID y páginas web de distintas OVI y asociaciones, hay que añadir gran número de blogs y páginas editadas por miembros del MVI que destacan por su gran dedicación, por su valor informativo y su carácter reivindicativo. A modo de ejemplo citamos https://vidaindependiente.org/, editada por Ismael Llorens Santamaría.

Un empeño especial del MVI en España ha sido la acción sociopolítica en torno a la normativa vigente y su diálogo directo con los representantes de los partidos políticos, encargados de llevar a cabo las políticas 
diseñadas para el colectivo de personas con diversidad. Quizá el ejemplo más importante de esta actividad sea el conjunto de acciones emprendidas por el FVID en torno a la Ley 39/2006 de Promoción de la Autonomía y atención a las personas en situación de dependencia, donde logró introducir la figura y prestación del asistente personal que debe precisarse como un derecho, y no como un medio de comercialización de servicios (FVID, 2017; García-Santesmases, 2012).

Después de aprobada la ley, ENIL y FVID llegaron, por ejemplo, a negociar con la Generalitat Valenciana un decreto de prestaciones que va más allá de los mínimos contemplados en la misma ley, aumentando las cuantías de prestación ${ }^{5}$. En 2015 el FVID elaboró un documento de propuesta política sobre la figura del asistente personal y su implantación en la sociedad española (Arnau et al., 2015) y en 2018 el FEVI en las conclusiones del segundo Congreso Europeo de Vida Independiente celebrado el Valencia los días 25 y 26 de noviembre presentó incluso una propuesta de ley orgánica sobre el asistente personal en España ${ }^{6}$.

Sin embargo, no hay en España muchas personas con discapacidad que trabajen en la política activa, quizá fruto de la desigualdad de oportunidades en formación académica y profesional, o, simplemente, como un síntoma más de la falta de integración social (Belzunegui y Puig, 2015). El CERMI, en referencia al art. 29 de la Convención de 2006, pide que haya una mayor participación política de personas con discapacidad en los partidos. El $\mathrm{MVI}$ es muy consciente de la gran influencia que ello significaría en el nuevo modo de percibir a las personas con discapacidad, a causa de su repercusión mediática y como medio de poder actuar directamente en el desarrollo de normativas inclusivas, así como en el control en la aplicación de las mismas (Cfr. Gálvez, 2017).

\subsection{Contexto}

Hay en España otras asociaciones de vida independiente, nacidas desde el mismo MVI y otras que poco a poco van incorporando sus principios a sus instituciones, como es el caso de las "Escuelas de Vida", promocionadas por Down España (Illán y Molina, 2013; Elkartu, 2018). Pero lo cierto es que siguen siendo una gran mayoría las asociaciones 'tradicionales', basadas en modelos asistenciales, apoyadas por la creencia de que este modelo, tanto a nivel nacional o local, les resulta más barato a las administraciones del Estado ${ }^{7}$, y con la confianza en que también pueden tener un mayor control sobre ellas.

El CERMI representa a más de 7000 asociaciones. Teniendo en cuenta además que no todas las asociaciones de este tipo están federadas en tales instituciones, se supone que habrá bastantes más. En esta clase de asociaciones más tradicionales se suelen ofertar diversos programas de rehabilitación, asesoramiento y gestión, etc., entre los cuales también figuran servicios de ayuda a domicilio. Sus servicios suelen ceñirse a las necesidades básicas dentro del hogar $\mathrm{y}$, excepcionalmente, a servicios de acompañamiento fuera del domicilio. Este tipo de servicios se pactan normalmente con el trabajador social y todo se gestiona desde la

5. Cfr. Arts. 15 y 23 de la Orden 5/2011, de 6 de junio, de la Conselleria de Bienestar Social, de modificación de los artículos 4 y concordantes de la Orden de 5 de diciembre de 2007, por la que se regulan los requisitos y condiciones de acceso a las ayudas económicas del Programa de Atención a las Personas y a sus Familias en el marco del Sistema para la Autonomía y Atención a la Dependencia en la Comunitat Valenciana [2011/6656], Diario oficial de la Comunidad Valenciana, núm. 6539 de 9 de junio de 2011, pp. 23597-23604.

6. Federación de Vida Independiente (FEVI) (2018): Proposición de ley orgánica reguladora de la asistencia personal para realizar el derecho humano fundamental de las personas con diversidad funcional a vivir en España de forma independiente y a ser incluido en la comunidad (Ley de Vida Independiente de las personas con diversidad funcional) (en línea) <https://drive.google.com/file/d/1VQAMG5zoyjopiA9i-8cLifeYx_YdpxUK/ view>, acceso 21 de enero 2019.

7. Lo cual no parece ser cierto, según se demostró desde los primeros estudios sobre el pago directo en el Reino Unido, promovido por el Comité de vida independiente (Evans, 2003: 204). 
asociación. Un ejemplo de este tipo asistencial es el programa de Fomento de Autonomía Personal (FAP) ofertado por COCEMFE-Valencia y cofinanciado con aportaciones de la administración autonómica, donaciones privadas y copago de los mismos usuarios en algunos casos (cuando la normativa lo exige). En el mismo sentido operan los servicios de ayuda a domicilio (SAD), el principal modelo promocionado por la mal llamada Ley de dependencia en España ${ }^{8}$.

Por otra parte, las asociaciones tradicionales están diseñadas frecuentemente con criterios de enfermedad y orientadas a la realización de actividades deportivas o rehabilitadoras, etc. Todavía son reflejo de una perspectiva médica/rehabilitadora, sin duda necesaria y complementaria, pero que no puede hacernos olvidar los derechos del colectivo integrado por personas dotadas de dignidad, que es donde pone el acento el MVI (Palacios y Romañach, 2006: 131).

También tenemos que advertir que en España se pueden encontrar, "centros de vida independiente" privados que, sin embargo, no pertenecen al movimiento. Suelen ser sociedades lucrativas que proponen el eslogan como medio para atraer clientes ${ }^{9}$.

\section{Conclusiones}

A pesar de que el MVI ha realizado en España una labor muy positiva de concienciación, la realidad es que sigue siendo, al menos cuantitativamente, un colectivo menor con respecto a las asociaciones tradicionales de personas con discapacidad. El hecho de que haya tan pocos asistentes personales en España (Carbonell, 2018) o que la presencia de personas con diversidad funcional en las instituciones políticas sea tan escasa, son claros síntomas de que falta mucho por recorrer para su inclusión social.

Sin embargo, a pesar del decaimiento generalizado del Estado social y los recortes presupuestarios en favor de una supuesta estabilidad económica que nunca llega, este movimiento sigue demostrando que está muy activo, como lo evidencia la celebración del II Congreso europeo celebrado en Valencia en 2018. Es necesario un impulso social que continúe luchando si no quiere sucumbir a un futuro distópico de injustica y desigualdad, donde no cabrían ya los mismos derechos humanos que concebimos como Naciones Unidas. Nuestra propuesta estaría dirigida en primer lugar a buscar la unión de fuerzas desde todas las entidades representativas como el CERMI, las miles de asociaciones y el mismo MVI. Seguidamente, se deberían centrar en lo que el CERMI (2017) denomina "constitucionalizar sus derechos". Los tratados internacionales, si los derechos allí reconocidos no se ven refrendados por las constituciones nacionales como derechos fundamentales y las leyes posteriores que los desarrollan, quedan sin suficientes garantías y, en consecuencia en la mayoría de ocasiones, vacíos de contenido (Cruz, 1989: 40; Gutiérrez, 2015: 269).

8. Ley 39/2006, de 14 de diciembre, de Promoción de la Autonomía Personal y Atención a las personas en situación de dependencia (LAPAD). 9. A propuesta de ENIL, desde el European Disability Forum (EDF) se denuncia el mal uso del término vida independiente por parte de algunos Estados y de asociaciones particulares interesadas en subvenciones, que no se ciñen a esta idea básica de vida independiente que propone el MVI. Cfr. en línea http://www.enil.eu/news/european-disability-movement-agrees-definition-of-independent-living/, acceso 21 de enero de 2019. Cfr. ENIL "Institutions Are Not Solutions: Learning from the Swedish experience". Audiovisual en línea <https://www.youtube.com/ watch?v=aFxYBZ2iXc4>, acceso 21 de enero de 2019. Cfr. www.cvi-bcn.org/es (Consulta, 24/09/2019). 


\section{Referencias bibliográficas}

Aparicio, M. (2016): “Estigmatización, invisibilización y cosificación de las personas con diversidad funcional. Una aproximación desde la justicia como reconocimiento de Axel Honneth”. Revista Española de Discapacidad, 4 (1): 177-190.

Arnau et al. (2015): La Asistencia Personal. Características y acciones a tomar para su implantación (en línea). <http:// forovidaindependiente.org/wp-content/uploads/2016/11/FVID-La_Asistencia_Personal-Caracteristicas_y_ acciones_a_tomar_para_su_implantacion.pdf>, acceso 21 de enero de 2019.

Bastida, F. J. (2010): “El entendimiento constitucional de la discapacidad”, en Presno, M. A. y Sarlet, I. W. (eds.): Los derechos sociales como instrumento de emancipación. Cizur Menor (Navarra): Thomson Reuters Aranzadi.

Belzunegui, A. y Puig, X. (2015): "Trayectorias educativas inacabadas: los problemas de los jóvenes con discapacidad para continuar estudios”. Sistema, 240: 81-100.

Bueno, J. R. y Villatoro, K. (2012): "De la discapacitat a la diversitat funcional: critiques fonamentals i presentación de noves perspectives". Futura, 23: 6-10.

Carbonell, G. J. (2018): La figura del asistente personal en el Movimiento de Vida Independiente: la experiencia en la Comunidad Valenciana. Tesis doctoral. UNED (en línea). <http://e-spacio.uned.es/fez/view/tesisuned:EDPg-DeryCSoc-Gjcarbonell>, acceso 20 de enero de 2019.

Centeno, A. et al. (2008): Indicadores de Vida Independiente (IVI) para la evaluación de políticas y acciones desarrolladas bajo la filosofía de Vida Independiente. Foro de Vida Independiente (en línea). <http://www.minusval2000.com/pdf/Indicadores_de_Vida_Independiente.pdf>, acceso 21 de enero de 2019.

Comité Español de Representantes de Personas con Discapacidad (CERMI) (2017): El CERMI pide al senado incluir los derechos sociales como fundamentales en la Constitución (en línea). <https://www.cermi.es/es/ actualidad/noticias/el-cermi-pide-en-el-senado-incluir-los-derechos-sociales-como-fundamentales-en>, acceso 21 de enero 2019.

Comunidad Valenciana. Orden 5/2011, de 6 de junio, de la Conselleria de Bienestar Social, de modificación de los artículos 4 y concordantes de la Orden de 5 de diciembre de 2007, por la que se regulan los requisitos y condiciones de acceso a las ayudas económicas del Programa de Atención a las Personas y a sus Familias en el marco del Sistema para la Autonomía y Atención a la Dependencia en la Comunitat Valenciana. [2011/6656], Diario oficial de la Comunidad Valenciana, 9 de junio de 2011, núm. 6539, pp. 23597-23604.

Cruz, P. (1989): "Formación y evolución de los derechos fundamentales". Revista Española de Derecho Constitucional, 9 (25): 35-62.

De Asís, R. (2016): "El eje de la accesibilidad y sus límites”. Anales de Derecho y Discapacidad, I (1): 51-68.

Elkartu (2018): Guía para el diseño de políticas inclusivas e intervenciones sociales orientadas al modelo de vida independiente en el ámbito de la discapacidad. Donostia-San Sebastián: Elkartu.

España. Ley 39/2006, de 14 de diciembre, de Promoción de la Autonomía Personal y Atención a las personas en situación de dependencia, Boletín Oficial del Estado, 15 de diciembre de 2006, núm. 299, pp. 44142-44156.

España. Ley 13/1982, de 7 de abril, de integración social de los minusválidos (LISMI), Boletín Oficial del Estado, 30 de abril de 1982, núm. 103, pp. 11106-11112. 
Evans, J. D. (2003): “El Movimiento de Vida Independiente en el Reino Unido", en García, J. V. (ed.): El Movimiento de Vida Independiente. Experiencias internacionales. Madrid: Fundación Luis Vives.

Federación de Vida Independiente (FEVI) (2018): Proposición de ley orgánica reguladora de la asistencia personal para realizar el derecho humano fundamental de las personas con diversidad funcional a vivir en España de forma independiente y a ser incluido en la comunidad (Ley de Vida Independiente de las personas con diversidad funcional) (en línea). <https://drive.google.com/file/d/1VQAMG5zoyjopiA9i-8cLifeYx_YdpxUK/view>, acceso 21 de enero de 2019.

Fernández, M. et al. (2016): Las TIC, una oportunidad para la inclusión. Barcelona: Observatorio de la Discapacidad Física. Serie Monográfico n. 7.

Ferreira, M. A. V. (2010): "De la minus-valía a la diversidad funcional: Un nuevo marco teórico-metodológico". Política y Sociedad, 47 (1): 45-65.

Finkelstein, V. (2001): The social Model of Disability Repossessed (en línea). <https://disability-studies.leeds.ac.uk/ wp-content/uploads/sites/40/library/finkelstein-The-Social-Model-of-Disability-and-the-Disability-Movement. pdf $>$, acceso 20 de enero de 2019.

Foro de Vida Independiente y Divertad (FVID) (2017): Archivo de la categoría: Asistencia Personal y LEPA (en línea). $<$ http://forovidaindependiente.org/category/general/mediateca/documentacion/asistencia-personal-y-lepa/>, acceso 21 enero de 2019.

Foro de Vida Independiente y Divertad (FVID) (2010): Nuestro compañero Patxi nos ha dejado (en línea). <http:// forovidaindependiente.org/nuestro-companero-patxi-nos-ha-dejado/>, acceso 21 de enero de 2019.

Gálvez, L. A. (2017): "El régimen de la participación electoral de las personas con discapacidad en los cuarenta años de democracia". Revista de Derecho Político, 100: 1057-1096.

García, J. V. (2003): El Movimiento de Vida Independiente. Experiencias internacionales. Madrid: Fundación Luis Vives.

García-Santesmases, A. (2012): La Operación Pozoblanco o la construcción de una identidad colectiva, FVID (en línea). <http://forovidaindependiente.org/la-operacion-pozoblanco-o-la-construccion-de-una-identidad-colectiva/>, acceso 21 de enero de 2019.

Gardien, È. (2010): “L'émergence en France des mouvements de pairs-représentants". Journal des anthropologues, 122-123: 87-114.

Gargarella, R. (1998): "Primeros apuntes para una teoría sobre los derechos sociales. ¿Es posible justificar un tratamiento jurídico diferenciado para los derechos sociales e individuales?". Jueces para la democracia, 31: 11-15.

Gómez, N. y Arroyo, J. (2013): “Oficinas de Vida Independiente”. Revista Latinoamericana de Educación Inclusiva, 7 (1): 79-52.

Gutiérrez I. (2015): Elementos de Derecho constitucional español. Madrid: Marcial Pons.

Hayashi, R. y Okuhira, M. (2008): "El movimiento por los derechos en el ámbito de la discapacidad en Japón. Pasado, presente y futuro", en Barton, L. (ed.): Superar las barreras de la discapacidad. 18 años de "Disability and Society". Madrid: Morata.

Honneth, A. (2014): El derecho de la libertad: esbozo de una eticidad democrática. Madrid: Katz. 
Illán, N. y Molina, J. (2013): "La Filosofía de Escuela de Vida. Sus aportes para la promoción de Vida Independiente en el colectivo de personas con discapacidad intelectual". Revista Latinoamericana de Educación Inclusiva, 7 (1): 23-38.

IPADEVI (2010): Declaración mundial de Compostela sobre la contribución de las personas con diversidad funcional (discapacidad) a una cultura de paz (en línea). <https://es.slideshare.net/solearnau/declaracin-mundialcompostela-2010>, acceso 21 de enero de 2019.

Jiménez-Martín, S. y Viola, A. (2017): Informe 2017 del Observatorio de Dependencia. Madrid, Fundación de Estudios de Economía Aplicada (FEDEA), Serie: Estudios sobre la Economía Española n. 2017/22.

Lasarte, C. (2010): Compendio de Derecho civil. Trabajo Social y Relaciones laborales. Madrid, Dykinson.

Lobato, M. (2005): Carta al FVID de propuesta del término diversidad funcional (en línea). <https://es.groups. yahoo.com/neo/groups/vidaindependiente/conversations/topics/9622>, acceso 22 de enero 2019.

López, A. y Gutiérrez, I. (2002): Elementos de derecho público. Madrid: Marciall Pons.

Maestro, G. y García, M. A. (1999): Marginación, Estado Social y Prestaciones Autonómicas. Barcelona: Cedecs.

Maraña, J. J. (2004): Vida Independiente. Nuevos modelos organizativos. Santiago de Compostela: AIES.

Maraña, J. J. y Lobato, M. (2003): “El Movimiento de Vida Independiente en España”, en García, J. V. (ed.): El Movimiento de Vida Independiente. Experiencias internacionales. Madrid: Fundación Luis Vives.

Martínez, K. (2003): “El Movimiento de Vida Independiente en Estados Unidos”, en García, J. V. (coord.): El Movimiento de Vida Independiente. Experiencias internacionales. Madrid: Fundación Luis Vives.

Miranda, J. (2016): "La accesibilidad universal y su gestión como elementos imprescindibles para el ejercicio de los derechos fundamentales". Anales de Derecho y Discapacidad, 1 (1): 89-108.

Moreno, F. J. y Del Pino, E. (2015): Desafíos del Estado de Bienestar en Noruega y España. Nuevas políticas para atender a nuevos riesgos sociales. Madrid: Tecnos.

Naciones Unidas (2006): Convención sobre los Derechos de las Personas con Discapacidad. Nueva York: ONU.

Nakanishi, S. (1997): "Historical Perspective and Development of Independent Living Movement in Japan". Disability Information Resources (DINF) (en línea) <http://www.dinf.ne.jp/doc/english/resource/z00009/z0000909. html>, acceso 20 de enero de 2019).

Palacios, J. y Romañach, J. (2006): El Modelo de la Diversidad. La Bioética y los Derechos Humanos como herramientas para alcanzar la plena dignidad en la diversidad funcional. Madrid: Ed. Diversitas-AIES.

Pisarelo, G. (2007): "Concepciones liberales y socialistas de los derechos sociales: una respuesta a Ricardo García Manrique". Anuario de la Filosofía del derecho, 25: 499-508.

Ratzka, A. D. (2003): "El movimiento de Vida Independiente en Suecia”, en García, J. V. (coord.): El Movimiento de Vida Independiente. Experiencias internacionales. Madrid: Fundación Luis Vives.

Ratzka, A. D. (1992): Towards an operational definition of Personal Assistance (en línea). <http://www.independentliving.org/toolsforpower/tools15.html>, acceso 20 de enero de 2019.

Ratzka, A.D. (1982): “Hemtservice - i vems regi?". Svenk Handikapptidskrift (en línea). <http://www.independentliving.org/docs3/ar1982.html>, acceso 20 de enero de 2019.

Romañach, J. (2009): Bioética al otro lado del espejo. La visión de las personas con diversidad funcional y el respeto a los derechos humanos. Santiago de Compostela: Diversitas Ediciones. 
Sanjosé, A. y Cardona, J. (2010): Informe sobre la inconstitucionalidad del supuesto de "aborto eugenésico" previsto en la Ley orgánica de salud sexual y reproductiva y de la interrupción voluntaria del embarazo (en línea). <http://www.minusval2000.com/pdf/fvi_aborto_inconsitucionalidad.pdf>, acceso 22 de enero de 2019.

Sennet, R. (2012): El respeto. Sobre la dignidad del hombre en un mundo de desigualdad. Barcelona: Anagrama.

Shakespeare, T. (2008): “La autoorganización de las personas con discapacidad: ¿Un nuevo movimiento social?”, en Barton, L. (ed.): Superar las barreras de la discapacidad. 18 años de "Disability and Society". Madrid: Morata.

Toboso, M. (2017): “Capacitismo”, en Platero, R. L. et al. (coords.): Barbarismos queer y otras esdrújulas. Barcelona: Bellaterra.

Urmeneta, X. (2012): "El binomio indispensable: asistencia personal y vida independiente. La experiencia de Gipuzkoa". Boletín del Observatorio Estatal de la Discapacidad (OED), 4: 54-68.

Urmeneta, X. (2011): Vida Independiente y Asistente Personal. La experiencia de Gipuzkoa (en línea). <http://www. forovidaindependiente.org/vida_independiente_y_asistencia_personal>, acceso 21 de enero de 2019. 